\title{
Aportes desde la bioarqueología al estudio de poblaciones urbanas de la Colonia en Sudamérica (Mendoza, Argentina)
}

\author{
Daniela Mansegosa \\ CONICET; FFyL, Universidad Nacional de Cuyo; CIRSF \\ mansegosad@yahoo.com.ar \\ Horacio CHIAvazzA \\ FFyL, Universidad Nacional Cuyo; CIRSF \\ hchiavazza@gmail.com
}

Recibido: 7 de enero de 2014

Aceptado: 7 de septiembre de 2015

\section{RESUMEN}

En el presente trabajo se dan a conocer los resultados de los análisis de los restos óseos humanos recuperados mediante intervenciones arqueológicas realizadas en el año 2009 por el Centro de Investigaciones Ruinas de San Francisco en el templo San Francisco, ubicado en el Norte de Mendoza. El sector excavado corresponde a la pilastra R ubicada en la entrada del templo del siglo XVIII, abarcando tanto el exterior como el interior del mismo. Se presentan los resultados de los estudios bioantropológicos y tafonómicos del conjunto óseo correspondiente a seis individuos articulados. También se define la cronología de cada individuo de acuerdo a su posición en el espacio y contexto de entierro. Estos estudios aportan información a la caracterización de la salud y nutrición de las poblaciones históricas urbanas de la ciudad de Mendoza.

Palabras clave: Estrés fisiológico, patologías, poblaciones históricas, Mendoza, Argentina.

\section{Bioarchaeological Contributions to the Study of Colonial Urban Populations in South America (Mendoza, Argentina)}

\begin{abstract}
In this paper we give the results of the analysis of human skeletal remains recovered by archaeological excavations carried out in 2009 by the Center for Research Ruins of San Francisco in San Francisco Temple, located in the north of Mendoza. The sector excavated R corresponds to the pilaster at the entrance of the temple of the eighteenth century, covering both the exterior and interior of the area. Bioanthropological and taphonomic results corresponding to six individuals from the whole bone. The chronology of each individual according to their position in space and burial context is also defined. These studies contribute to the characterization of the health and nutrition of urban historical population in the city of Mendoza.
\end{abstract}

Key words: Physiological stress, diseases, historical populations, Mendoza, Argentina.

Sumario: 1. Introducción. 2. Materiales y métodos. 3. Resultados. 4. Discusión. 5. Conclusiones. 6. Referencias bibliográficas.

\section{Introducción}

Los templos coloniales de América ofrecen inmejorables condiciones para obtener una caracterización de la población colonial en sentido amplio. En el caso de Mendo$\mathrm{za}$, fundada en $1561 \mathrm{y}$ con evidencias documentadas y arqueológicas de contactos por lo menos desde 1541 - con la fundación de Santiago de Chile-(Chiavazza y Mafferra 2007), la ciudad fue consolidándose y dando lugar a la instalación de numerosas órdenes religiosas. Estas ocuparon lugares centrales del plan urbano. Allí generaron 


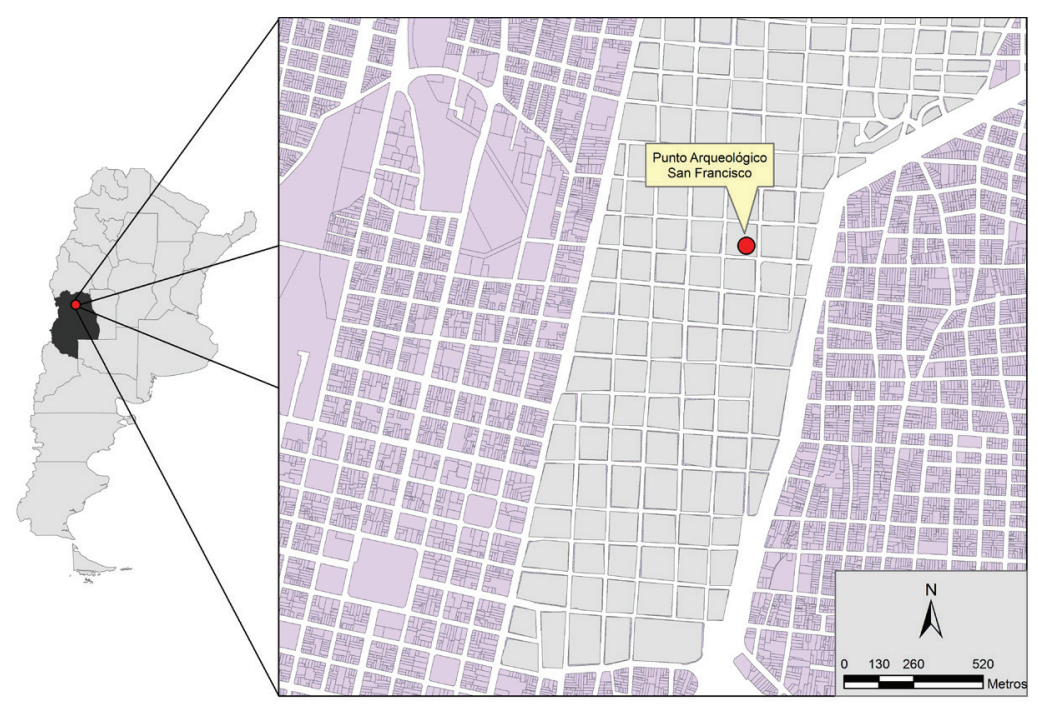

Figura 1: Localización de las ruinas de San Francisco dentro del Área Fundacional (superficie gris), provincia de Mendoza, Argentina.

entre dos y tres pulsos de crecimiento edilicio, pasando de templos y dependencias modestas a grandes construcciones en ladrillo de buena factura y destacado orden arquitectural en el contexto de una ciudad que no se destacaba por sus edificios. Así, los templos, desde un punto de vista heurístico, pueden considerarse puntos con valiosa concentración informativa para explicar procesos sociales desde el punto de vista de las condiciones de vida de los ciudadanos (Chiavazza 2008).

Desde el punto de vista histórico está profusamente documentado el rol que tuvieron las órdenes religiosas en el manejo de la «buena muerte», haciéndose cargo de los difuntos a cambio de cuotas y pagos por parte de los familiares, en muchos casos con parte de lo heredado. En esta función se desarrollaban ritos, generalmente con escasa marca material, pero que terminaban en acciones con fuerte carga espacial. Así, las personas eran enterradas de acuerdo a su voluntad y posibilidad económica en determinados sectores del espacio sacro. Este se valuaba con mayor o menor precio conforme se estuviera dentro o fuera del edificio. Incluso, dentro del templo, existían sectores más valorados (por ej. cerca de la pila de agua bendita) (Chiavazza 2005). Así, desde el punto de vista arqueológico, la excavación de cuerpos sin mayores referencias en las tumbas acerca de su rol y riqueza en la sociedad, adquiere un valor fundamental el análisis de posicionamientos. No obstante, como dijimos, en Mendoza, gran parte de los edificios se construyeron y reconstruyeron, dando lugar a procesos distorsivos respecto a la integridad del registro bioantropológico. De este modo, al establecer las secuencias constructivas y las relaciones de estratificación y distribución, se lograron avances en la atribución de cuerpos a diferentes etapas de la historia de los complejos arquitectónicos, pudiendo atribuir la localización de los cuerpos a sectores con distintas significaciones. Tales significados son una clave para desarrollar hipótesis referidas a las diferencias sociales establecidas en términos materiales de existencia. De la vida a la muerte y de esta al entierro. Justamente la 


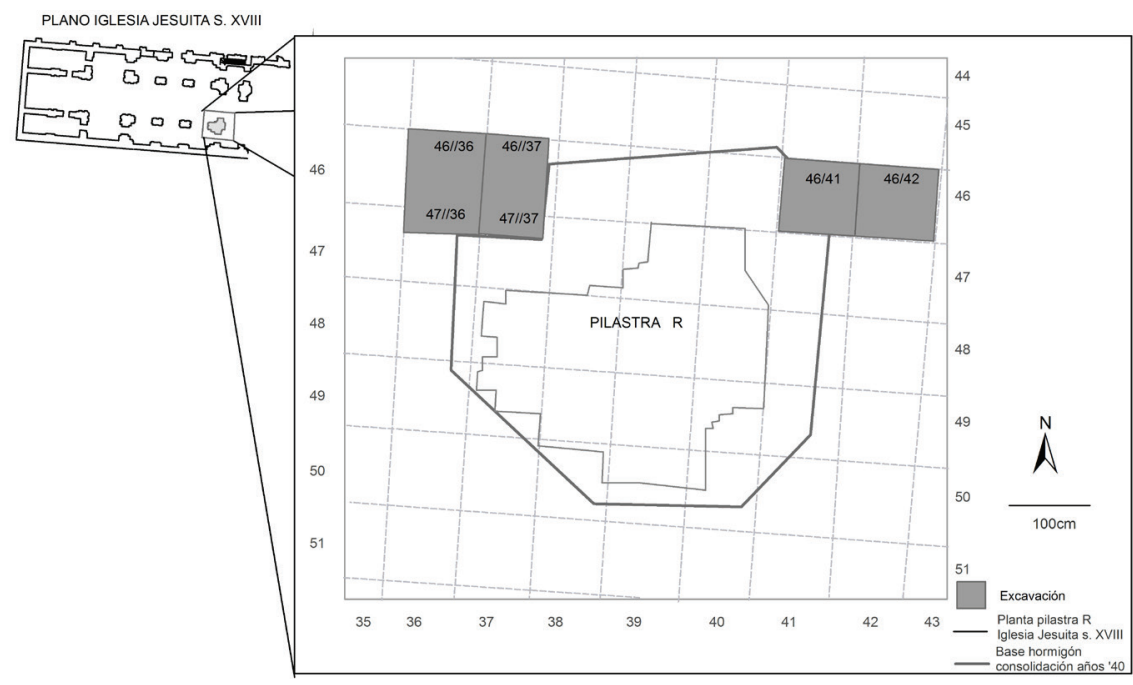

Figura 2: Localización de las excavaciones en las ruinas de San Francisco.

ubicación de este daría cuenta de la relativa posición socio-económica del difunto en su vida.

Expondremos el resultado de excavaciones llevadas a cabo en el contexto de seguimiento de obras de consolidación de las conocidas como «Ruinas de San Francisco» de la ciudad de Mendoza. Enfatizaremos el estudio bioantropológico de los restos recuperados, los cuales se integran con otros obtenidos precedentemente y oportunamente publicados (Chiavazza 2005) (Figura 1).

\section{Materiales y métodos}

Se excavaron cuatro cuadrículas de $1 \mathrm{~m}^{2}$ y dos cuadrículas de 0,20 x $1 \mathrm{~m}$, con una profundidad de $2 \mathrm{~m}$, empleando niveles artificiales de extracción de $5 \mathrm{~cm}$ y respetando las capas naturales. Se alcanzó un total de $4,40 \mathrm{~m}^{2}$ de superficie excavada; en la Figura 2 se observa la estructura del templo San Francisco y la localización de las cuadrículas excavadas. El sedimento extraído se tamizó en una zaranda con una malla de $2 \mathrm{~mm}$. Se siguió un relevamiento detallado de la ubicación y disposición de los esqueletos. El tipo de sedimento, limo arcilloso semicompacto, permitió la fácil excavación y extracción de los mismos. Dado el relativo estado de conservación de los huesos, fue necesario aplicarle primal in situ a los más deteriorados para su consolidación.

Se hallaron dos tipos de contextos. Por un lado los entierros que se mantienen en posición primaria, que suman un total de 6 individuos. Estos manifiestan niveles de integridad que permiten asegurar la correlación entre lugar de depósito y lugar de hallazgo. Por otra parte, están los contextos secundarios, que comprenden un NMI de 2. Estos corresponden a huesos mezclados o partes de cuerpos con bajo nivel de 
integridad y que responden a patrones de desestructuración producidos por diversas razones (corrimientos por re-excavación de fosas, excavación de obras de edificación e incluso procesos posteriores al derrumbe producido por el terremoto del 20 de marzo de 1861). Así, la muestra total objeto de este análisis es de 8 individuos.

En los individuos articulados adultos se determinó el sexo a partir de los indicadores morfológicos del cráneo y de la pelvis siguiendo las consideraciones de Buikstra y Ubelaker (1994), además se evaluó el dimorfismo a partir de los diámetros de la cabeza del húmero y fémur siguiendo las fórmulas establecidas por Stewart (1970, en Byers 2002) y Pearson (1917-1919, en Bass 1987) respectivamente. La edad en adultos, se obtuvo a partir del análisis del estado de obliteración de las suturas craneales (Meindl y Lovejoy 1985), cambios en la morfología de la superficie auricular del ilion (Lovejoy et al. 1985) y modificaciones en la superficie de la sínfisis púbica (Todd 1921). En subadultos el sexo se estimó en base a las características del ílion (Schutkowski 1993; Weaver 1980). La edad se calculó a partir del desarrollo y erupción dentaria (Ubelaker 1989), y de las longitudes de los huesos largos de las extremidades y clavículas (Buikstra y Ubelaker 1994). Los cálculos de las edades según los datos continuos se establecieron a partir de los parámetros desarrollados por Scheuer y Black (2004).

En el caso de huesos correspondientes a entierros secundarios el NMI se calculó en base a la cantidad de fémures y húmeros, teniendo en cuenta edad, sexo, lateralidad, longitud y robusticidad. Se pudo establecer el sexo a partir de los diámetros de la cabeza de estos elementos siguiendo la metodología mencionada. La confiabilidad de estas variables para la determinación sexual ha sido testada en muestras control del área de estudio con resultados positivos (Mansegosa 2010; Mansegosa y Giannotti 2012). La edad en estos conjuntos se clasificó en dos grandes grupos, adultos y subadultos, y dentro de éste último se siguió la metodología mencionada arriba. La longitud y robusticidad se midieron en base a las variables métricas detalladas por Buikstra y Ubelaker (1994); cuando eran diferentes de forma significativa se consideraron que correspondían a distintos individuos.

Por otra parte, se evaluó el estado de completitud y conservación de cada uno de los individuos, para poder comparar con otras muestras y tener una perspectiva regional acerca de las modificaciones tafonómicas de naturaleza antrópica y no antrópica de los entierros en estos templos. De este modo se busca establecer comparaciones más precisas entre los bioindicadores evaluados (Mansegosa 2010). Para diagnosticar la integridad de cada individuo se describieron las unidades anatómicas presentes y se evaluó el grado de completitud de cada elemento. Esto último se realizó a partir de una escala creciente de 1 a 4, donde el grado 1 representa la presencia de menos del $25 \%$ del elemento, el grado 2 de 25 a $50 \%$, el grado 3 de 50 a $75 \%$ y el grado 4 de 75 a 100\%. Por otra parte, también se observó la presencia/ausencia de manchas de manganeso, depósito de carbonato de calcio, teñido/tinción de la superficie ósea, marcas de roedor, marcas de carnívoros e improntas de raíces con el fin de chequear factores actuantes en la conformación del registro (Barrientos et al. 2007).

En el conjunto de individuos articulados se evaluó la presencia/ausencia de indicadores inespecíficos de estrés nutricional e infeccioso (hipoplasias del esmalte dental, hiperostosis porótica), procesos infecciosos a nivel óseo y periodontal (periostitis, 
periodontitis, caries, abscesos y pérdida de dientes antemortem), indicadores de adecuación fisiológica de la cavidad oral (grado y patrón de desgaste dental) y lesiones traumáticas (Buikstra y Ubelaker 1994; Hillson 1996; Larsen 1997; Mensforth 1991; Ortner y Putschar 1981, entre otros).

Las hipoplasias de esmalte, se relevaron siguiendo la metodología desarrollada por Barrientos (1999), se analizaron en cada individuo los incisivos centrales, incisivos laterales, caninos y premolares, del maxilar y de la mandíbula, tanto izquierdos como derechos. Se observaron las superficies de las coronas de forma macroscópica, y se midieron con calibre digital con una resolución de $0.01 \mathrm{~mm}$. Se calculó el Índice de Simetría (IS) para discriminar entre hipoplasias aisladas resultado de causas no sistémicas (i.e. trauma) de aquellas producidas por procesos sistémicos (i.e. estrés ambiental). Para comparar las prevalencias de hipoplasia de esmalte entre individuos, se calculó el Número Mínimo de Eventos de Detención del Crecimiento Dental (NMSDCD). Por último se calculó la edad etiológica o de formación para todas las líneas de hipoplasias de esmalte (en adelante HE) detectadas mediante las fórmulas propuestas por Goodman y Rose (1991), y se estableció para cada individuo el Rango de Edades (RE) entre las cuales sufrió eventos de estrés, teniendo en cuenta las edades mínimas y máximas arrojadas por pares de hipoplasias simétricas.

\section{Resultados}

La muestra se estableció como compuesta por un total de ocho individuos de los cuales seis estaban articulados y el resto corresponde a los conjuntos desagregados. Dentro de los articulados, cuatro son adultos (tres masculinos y uno femenino), dos son infantiles (uno masculino y otro indeterminado) (Cuadro 1). En los conjuntos desarticulados el elemento diagnóstico más representado para determinar el NMI fue el fémur, en base a éste se calculó un NMI de dos individuos, de los cuales uno es adulto de sexo indeterminado debido a que la cabeza del fémur no estaba conservada y el otro es subadulto con una edad aproximada de 2 a 5 años.

Todos los individuos se encontraban orientados con la cabeza hacia el este y los pies hacia el oeste, en dirección paralela al templo. En todos los casos la posición del cuerpo era extendida de cúbito dorsal con los brazos flexionados hacia el abdomen. No se encontraron evidencias de cajón (i.e. clavos o madera), ni de ajuar como en otros casos (Chiavazza 2005).

El individuo NCPilR 1 es el que presenta la menor profundidad $(140-150 \mathrm{~cm}$ del nivel de piso actual) y es el único que tenía restos de escombros en la fosa, por lo que ha sido interpretado como el único individuo de esta muestra que fue enterrado en el templo del siglo XVIII, y este sector corresponde al interior del edificio (nave central).

Por otra parte, el individuo NCPilR 5 (a 195-198 cm de profundidad) probablemente sea el más antiguo de la muestra, ya que fue impactado cuando se cavó la fosa para el individuo NCPilR 6 (193-198 cm de profundidad), que fue inhumado al costado del mismo, quedando casi en contacto entre sí. Ambos se encontraban cortados por el cimiento del templo del siglo XVIII (edificado entre 1716-1733). El individuo 
Cuadro 1: Esqueletos articulados: sexo, edad de muerte, sector de entierro y cronología aproximada

\begin{tabular}{|c|c|c|c|c|c|c|}
\hline Individuo & Sexo & $\begin{array}{l}\text { Edad } \\
\text { (años) }\end{array}$ & $\begin{array}{c}\text { Sector de } \\
\text { entierro }\end{array}$ & $\begin{array}{l}\text { Cronología } \\
\text { (aprox.) }\end{array}$ & Cuadrículas & $\begin{array}{l}\text { Profundidad } \\
(\mathrm{cm})\end{array}$ \\
\hline NCPilR 1 & masculino & $40-45$ & $\begin{array}{l}\text { Interior } \\
\text { templo S. XVIII }\end{array}$ & XVIII & $\begin{array}{l}46 / 35 \\
46 / 36\end{array}$ & $\begin{array}{l}140 \\
150\end{array}$ \\
\hline NCPilR 2 & indetermin. & $10-12$ & $\begin{array}{l}\text { Exterior } \\
\text { Templo S. XVII }\end{array}$ & XVII & $\begin{array}{l}46 / 41 \\
46 / 42\end{array}$ & $\begin{array}{l}165 \\
180\end{array}$ \\
\hline NCPilR 3 & masculino & $>21$ & & & $\begin{array}{l}47 / 37 \\
46 / 37\end{array}$ & $\begin{array}{l}185 \\
198\end{array}$ \\
\hline NCPilR 4 & masculino & $12,5-13,5$ & & & $46 / 41$ & $\begin{array}{l}175 \\
184\end{array}$ \\
\hline NCPilR 5 & femenino & $21-30$ & & & $\begin{array}{l}46 / 42 \\
46 / 41\end{array}$ & $\begin{array}{l}195 \\
198\end{array}$ \\
\hline NCPilR 6 & masculino & $20-35$ & & & $\begin{array}{l}46 / 41 \\
46 / 42 \\
\end{array}$ & $\begin{array}{l}193 \\
198\end{array}$ \\
\hline
\end{tabular}

Cuadro 2: Estado de conservación e integridad de las muestras

\begin{tabular}{lllllll}
\hline \multirow{2}{*}{ Individuo } & \multicolumn{7}{c}{ Completitud (\%) } & \multirow{2}{*}{$\mathbf{M n O}_{\mathbf{2}}(\mathbf{\%})$} & \multirow{2}{*}{ Marcas antiguas } \\
\cline { 2 - 5 } & $\mathbf{1}$ & $\mathbf{2}$ & $\mathbf{3}$ & $\mathbf{4}$ & & \\
\hline NCPilR 1 & - & 6 & 4 & 90 & 2 & \\
NCPilR 2 & - & - & 24 & 76 & 45 & 1 húmero \\
NCPilR 3 & - & 1 & 28 & 71 & 44 & 1 fémur \\
NCPilR 4 & - & 22 & 39 & 39 & 35 & 1 fémur \\
NCPilR 5 & - & 10 & 41 & 49 & 62 & \\
NCPilR 6 & 1 & 10 & 25 & 64 & 63 & \\
\hline
\end{tabular}

NCPilR 4 hallado en las mismas cuadrículas, a unos pocos centímetros por encima de estos (175-184 cm de profundidad), fue enterrado algún tiempo después, ya que para su inhumación se impactaron los entierros precedentes. El último individuo inhumado en estas cuadrículas es NCPilR 2 (165-180 cm de profundidad), el cual presentaba asociados fragmentos de cerámica roja (probablemente del periodo correspondiente al siglo XVI, previo a la instalación de la iglesia en el lugar). El NCPilR 3 fue recuperado en otras cuadrículas pero a similar profundidad. Dada la profundidad de estos entierros, entre los 165-198 cm, y sector de los mismos, han sido atribuidos a entierros realizados en el exterior del templo del siglo XVII (como los casos de NCPilR 2 NCPilR 5 y NCPilR 6) (ver Cuadro 1).

Los análisis de integridad y conservación de la muestra, como así también de los bioindicadores de salud y nutrición se efectuaron sobre los esqueletos articulados, tanto en los adultos como en los subadultos. En los individuos subadultos, el NCPilR 4 presentaba sólo el esqueleto postcraneal, el cráneo estaba ausente por remoción postmortem debido a las intervenciones para la inhumación de nuevos cuerpos. El individuo NCPilR 2 se encontraba completo.

Entre los adultos los individuos NCPilR 1 y NCPilR 3 se encontraban completos exceptuando el húmero y radio en el primero. A NCPilR 5 le faltaban ambos brazos y la pierna izquierda. El NCPilR6 no presentaba el cráneo, sí la mandíbula, y registraba ausencia de tibia, peroné y pie izquierdo, el resto del esqueleto se encontraba presente. 


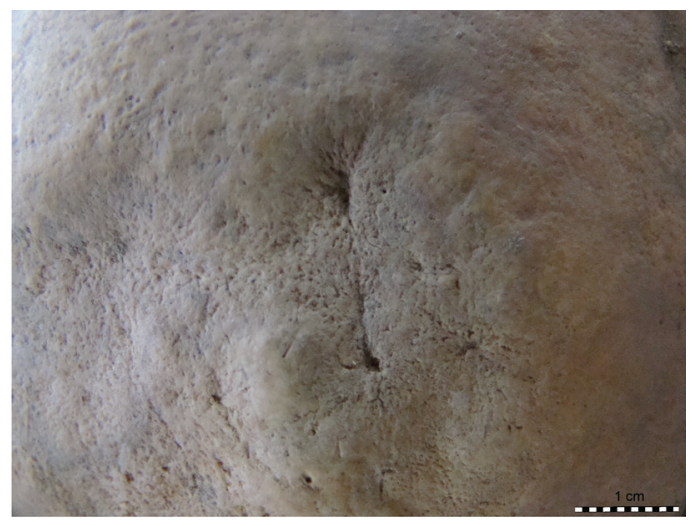

Figura 3: Cráneo del individuo NCPilR 1 con evidencias de treponematosis (frontal con osteoperiostitis remodelada).

Figura 4: Fémur derecho del individuo NCPilR 1 con lesiones atribuibles a treponematosis (sector medio y distal de la diáfisis).

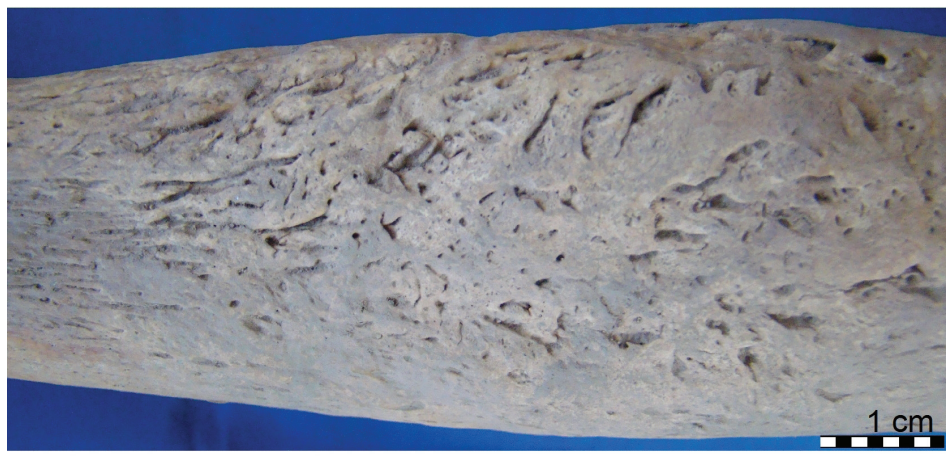

El grado de completitud de la mayoría de los casos mencionados, presentaban más del 50\% del elemento. En términos generales interpretamos que la muestra se halló en un estado de conservación e integridad medio (Cuadro 2). Por lo tanto, la resolución para la observación de patologías y traumas es media en todo el conjunto.

En cuanto a las alteraciones de la superficie ósea, se observó que el factor de mayor incidencia fueron los depósitos de manganeso. Estos afectaron entre un 35\% y $63 \%$ de los elementos de la muestra, exceptuando el esqueleto NCPilR 1 que mostró una incidencia del 2\%. En general el patrón de depósitos de manganeso observado es puntiforme de intensidad leve, con una distribución heterogénea. No se detectaron depósitos de carbonato de calcio, como tampoco improntas de raíces, ni marcas de roedores ni carnívoros. Se hallaron marcas antiguas en las diáfisis de huesos largos de tres individuos -en el individuo NCPilR 4 y NCPilR 3 en el fémur y en el NCPilR 2 en el húmero-; probablemente se deban a impactos producidos mediante los procesos de apertura de fosas para el entierro de nuevos individuos.

Para evaluar la presencia de estrés fisiológico se seleccionaron aquellos individuos que presentaban el cráneo. En tres (75\%) de los cuatro analizados se hallaron lesiones atribuibles a hiperostosis porótica, las cuales se encontraban activas en el individuo subadulto, mientras que en los adultos estaban remodeladas.

En cuanto a los procesos paleopatológicos, el 50\% de los individuos no presentaron ningún tipo de evidencia; en el otro $50 \%$ se hallaron lesiones atribuibles a algún tipo de proceso infeccioso. 
Cuadro 3: Patologías

\begin{tabular}{lll}
\hline Individuo & Hiperostosis porótica & Procesos infecciosos \\
\hline NCPilR 1 & No & Treponematosis \\
NCPilR 2 & Si (activa) & Periostitis \\
NCPilR 3 & Si (remodelada) & Periostitis \\
NCPilR 4 & Inobservable & No \\
NCPilR 5 & Si (remodelada) & No \\
NCPilR 6 & Inobservable & No \\
\hline
\end{tabular}

Cuadro 4: Detalle de indicadores de salud bucal por individuo

\begin{tabular}{llcccccccc}
\hline Individuo & N & PAM & PPM & AAN & D & Tipo de desgaste & C & S & HDE \\
\hline NCPilR 2 & 26 & 0 & 0 & 0 & 1 & Natural & 0 & 4 & 10 \\
NCPilR 3 & 13 & 2 & 1 & 13 & 4 & Plano & 0 & 5 & 8 \\
NCPilR 4 & 7 & 16 & 9 & 0 & 6 & Plano & 3 & 0 & 2 \\
NCPilR 5 & 23 & 0 & 6 & 1 & 4 & Plano & 0 & 3 & 9 \\
NCPilR 6 & 9 & 0 & 1 & 19 & 5 & Plano & 0 & 0 & 3 \\
\hline Total & 78 & 18 & 17 & 33 & & & 3 & 8 & 22 \\
\hline
\end{tabular}

$\mathrm{N}$ : total piezas dentarias presentes. PAM: pérdida antemortem. PPM: pérdida postmortem. AAN: ausente con hueso alveolar no asociado. D: promedio de desgate. C: total de dientes con caries. S: total de dientes con sarro. HDE: total de dientes con HDE

El caso del individuo NCPilR 1, presenta lesiones características de teponematosis, las cuales se distribuían en el cráneo, ambos fémures y tibias. El cráneo presentaba en el frontal osteoperiostitis gummatosa completamente remodelada; se observa como un sector deprimido con una cicatriz esclerótica; esta lesión es denominada «caries sicca» diagnóstica de la treponematosis (Figura 3). Los fémures presentaban periostitis gummatosa con una distribución simétrica a nivel de la mitad y tercio distal de la diáfisis, las lesiones mostraban un considerable grado de curación esclerótica, esta neoformación ósea provocó una ampliación de la corteza del hueso (Figura 4). Las tibias también presentaban periostitis incipiente en el tercio proximal de la diáfisis. No se registraron traumas en ninguno de los individuos.

El caso del individuo NCPilR 2 presentaba periostitis incipiente activa en ambas tibias a nivel de la diáfisis. El NCPilR 3 tiene ambas tibias con periostitis a lo largo de la diáfisis, presentan neoformación de hueso reactivo con una disposición de tipo laminar con estriaciones, probablemente resultado de una reacción secundaria a una infección. En el Cuadro 3 se detalla por individuo la presencia ausencia de hiperostosis porótica y procesos infecciosos.

En cuanto a la salud bucal (Cuadro 4) casi la totalidad de los individuos pudieron ser evaluados, exceptuando el NCPilR 4; todos ellos tenían piezas dentarias presentes y estaban relativamente bien representados, analizándose un total de 78 piezas dentarias. La mayoría de los individuos presentaba pérdida de elementos postmortem debido a factores postdepositacionales. Las pérdidas premortem se hallaron sólo en dos individuos, siendo NCPilR 1 el que presentó la mayor cantidad de alveolos reabsorbidos, tanto del maxilar como de la mandíbula, lo que se vincula con su avanzada edad. Este individuo es el único que registra caries que se encontraban en la raíz del 2 PM izquierdo superior y en el sector cervical interproximal del canino izquierdo e incisivo derecho de la mandíbula. 
Cuadro 5: Características relevadas de las HE registrada en la muestra.

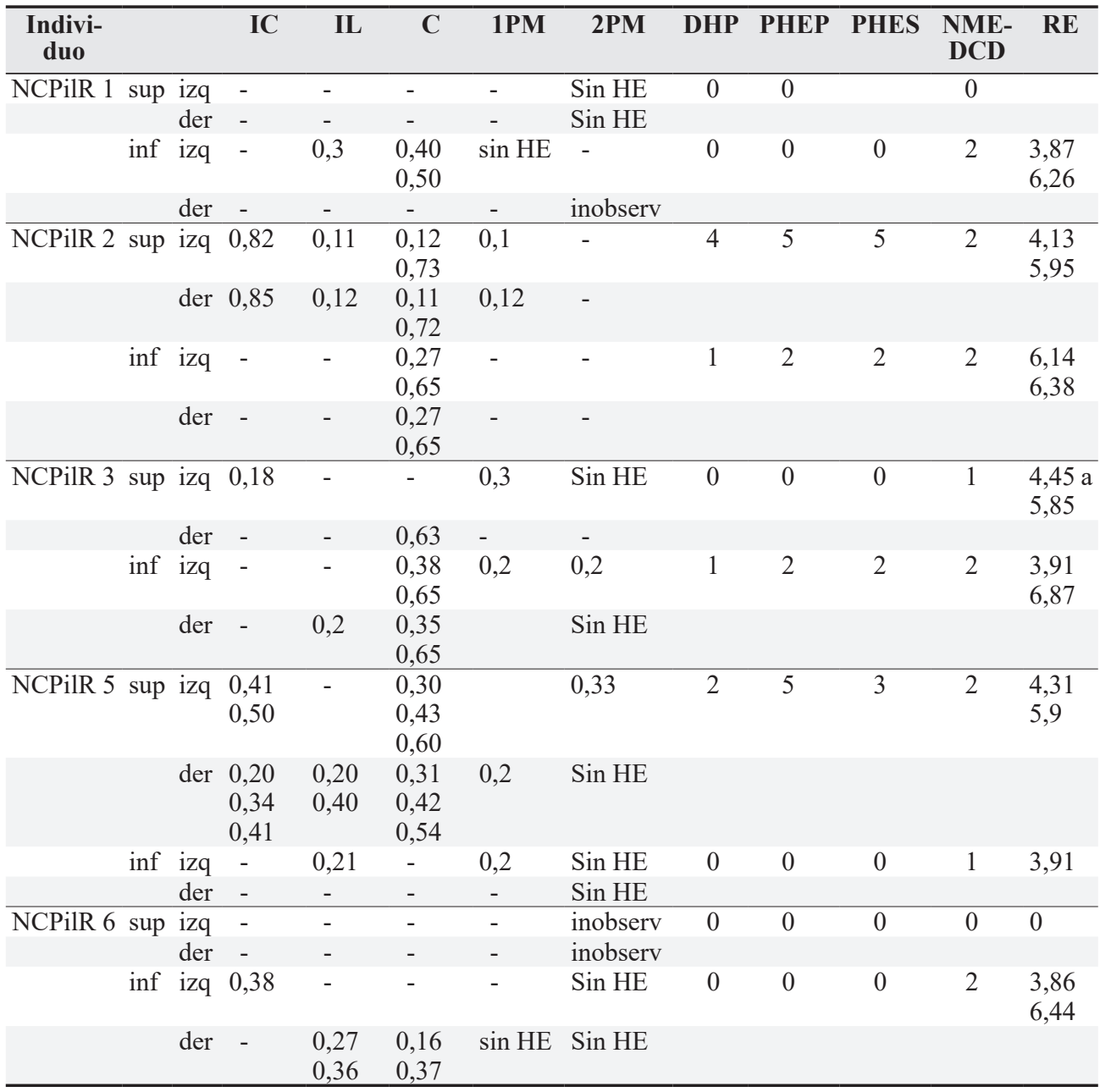

IC: incisivo central; IL: incisivo lateral; C: canino; 1PM: primer premolar; 2PM: segundo premolar; DHP: dientes homólogos posibles; PHEP: pares de hipoplasias posibles; PHES: pares de hipoplasias simétricas; NMEDCD: Número mínimo de eventos de detención del crecimiento dental. RE: rango de edades entre las cuales ocurrieron los episodios de estrés (años).

En general, en los individuos adultos se observa un grado de desgaste medio, vinculado con la edad de muerte; en el individuo subadulto el desgaste es mínimo; en todos los casos el tipo de desgaste es plano. Es significativa la presencia de hipoplasias de esmalte que se encontraron en todos los individuos analizados, lo cual se asocia con episodios de estrés fisiológico.

En cuanto a las hipoplasias de esmalte relevadas en cada uno de los individuos, del total de piezas observadas, 47 tenían posibilidades de presentar evidencias por ser incisivos, caninos o premolares (Cuadro 5). De éstos, 3 debieron ser descartados por el mal estado de conservación, quedando 44 piezas con potencialidad para observar 
líneas de hipoplasia. Todos los individuos observados presentaron al menos una línea de hipoplasia en alguno de los dientes observados, por lo que no se observan diferencias en la distribución según sexo ni edad.

El IS total de la muestra calculado fue de $86 \%$, lo cual indicaría una probabilidad bastante alta de que los casos de hipoplasia hayan ocurrido por procesos sistémicos. El cálculo del NMEDCD de la colección indica que todos los individuos presentaron al menos 2 eventos hipoplásicos. Los RE calculados de aparición de estos episodios de estrés están comprendidos entre los 3,86 a 6,87 años de edad, es decir en el límite entre la infancia y la niñez.

\section{Discusión}

A partir de la excavación y estudio del sitio, se reconoció que 5 individuos fueron enterrados en el exterior del templo del siglo XVII, los que presentan mayores profundidades han sido perturbados para una reutilización del sector, lo que provocó la remoción de algunas unidades anatómicas. Un único individuo se reconoció como inhumado en el interior de la iglesia del siglo XVIII. No se hallaron evidencias de cajón ni de ajuar asociado, lo cual es esperable para entierros de personas más pobres como lo plantea el modelo propuesto (Chiavazza 2005).

La completitud resultó diferencial, ya que todos registraban ausencia de algún elemento debido a la remoción antrópica y a los impactos producidos por las ampliaciones edilicias. Por otra parte, la conservación e integridad de los elementos era media, porque presentaban un alto grado de fragmentación, lo cual dificultó en algunos casos el análisis paleopatológico. Estas características referentes a la conservación e integridad de los entierros, son comunes a otros templos coloniales del Área Fundacional como es el caso de las excavaciones previas en este mismo templo (Chiavazza 2005) o los de San Agustín, Santo Domingo, La Merced y La Caridad. En el último caso, el grado de fracturas postdepositacionales y de pérdida ósea superaban el $50 \%$ de los elementos óseos de la muestra (Mansegosa 2010).

Estas características de los registros funerarios de la ciudad han sido interpretadas como resultado de la combinación de procesos antrópicos tales como, las prácticas de inhumación (depositación, reubicación, reentierro) y de los factores naturales tanto intrínsecos de los huesos como extrínsecos (actividad microbiana, acción de raíces, depósito de carbonato, entre otros) que afectaron la disposición, conservación e integridad de los elementos (Chiavazza 2005; Mansegosa 2010).

Es importante remarcar que los individuos correspondientes al exterior del templo del siglo XVII son jóvenes, uno es un juvenil y el resto adultos jóvenes, estos rangos de edad tienen baja mortalidad en los perfiles calculados para el Área Fundacional (entre $0-10 \%$ de la mortalidad total respectivamente, Mansegosa et al. 2013). Si bien las evidencias de procesos infecciosos se hallaron en dos de estos individuos, podemos pensar que el resto puede haber muerto a causa de algún tipo de enfermedad fulminante (i.e viruela documentada historiográficamente, Semorile et al. 1988), debido a que no presentaron evidencias de trauma. 
En referencia a las evidencias de hiperostosis porótica, se encontró un porcentaje alto de individuos con estas lesiones (75\%); todos corresponden al exterior del templo, es decir a las personas con menos recursos socio-económicos. El individuo juvenil presentaba este proceso activo al momento de la muerte, mientras que los adultos habrían superado la situación. Este podría ser un indicador de anemia, principalmente del tipo megaloblástica (Walker et al. 2009). La expansión medular del diploe, deriva en las lesiones tipo cribado características de la hiperostosis porótica. Lo cual es resultado de una reacción compensatoria del cuerpo para producir mayor cantidad de glóbulos rojos. Esto, no se produce en individuos con reservas de hierro agotadas y sí en individuos con carencia de la vitamina B12 y/o ácido fólico, característicos de las anemias megaloblástica y hemolítica. Las bajas cantidades de estas vitaminas pueden tener causas múltiples como la mala absorción, dieta pobre en productos derivados de animales, infecciones reiteradas, parásitos y enfermedades gastrointestinales (Walker et al. 2009).

Podemos interpretar que estas personas sufrieron procesos infecciosos (dos de los tres individuos que tenía hiperostosis porótica tenían evidencias de infecciones) y probablemente algún tipo de deficiencia nutricional, que podrían haber desencadenado la anemia o agudizarla si es que ya existía. Ello indica las difíciles condiciones de salud que experimentaron las personas de los estratos sociales con menos recursos, y la dificultad de sobrevivir.

Se detectó que los procesos infecciosos registrados afectaron a tres individuos. Dos eran casos de patologías infecciosas inespecíficas que afectaron a un joven y a un adulto, en ambos, el proceso estaba activo al momento de la muerte. Desde la historia, para Mendoza, se han documentado tempranas epidemias como la viruela. También hay menciones a fiebres no especificadas y a «pestes o males» (Semorile et al. 1988), los cuales están alertando sobre algún tipo de enfermedad infecto-contagiosa entre estas poblaciones.

El tercero, es el individuo que presentaba treponematosis, el cual constituye el primer caso arqueológico registrado en la provincia con esta patología. Este individuo ha sido asignado a los entierros del siglo XVIII por lo tanto es probable que haya vivido durante el siglo XVII. Su ubicación en un sector destinado al entierro para las personas con mayores recursos resulta consistente con una enfermedad que para dejar su marca en el esqueleto requiere entre 5 y 10 años de padecerla, interpretamos que ha sido una persona que ha tenido recursos como para mantenerse en el tiempo con esta enfermedad, es decir que le han proporcionado alimentos y ciertos cuidados que le permitieron sobrevivir durante un tiempo. Las manifestaciones de esta enfermedad dependen tanto de las condiciones inmunes del huésped humano (i.e. edad, sexo, adecuación de la dieta, tratamiento) como de las características del patógeno (biología, reacción a la respuesta inmune, estrategia reproductiva) y de la relación establecida entre ambos (flexible, agresiva) (Ortner 2003).

Como dato a tener en consideración (teniendo en cuenta las distancias temporales) debemos decir que en los primeros registros de higienistas realizados en la ciudad de Mendoza, hay estadísticas de mortalidad por sífilis. Se menciona que entre los años 1860 y 1896 (unos 200 años después del registro aquí analizado) hubo 105 defunciones, de las cuales 55 corresponden a infantes (Coni 1897: 229). Esto hace 
suponer que los 55 casos corresponden a sífilis congénita, infantiles que nacen con la enfermedad transmitida a través de placenta de la madre infectada. La sífilis fue una enfermedad históricamente incurable, mutilante y mal vista socialmente (Morales et al. 2004: 494). El aislamiento fue una de las medidas terapéuticas implementadas (Semorile et al. 1988) y una de las acciones sociales de la época que condenaban y aislaban a determinadas personas. En el caso de este individuo suponemos que no estuvo aislado, ya que le brindaron alimento y los cuidados necesarios para vivir con la enfermedad.

La evidencia de hipoplasia de esmalte se da en todos los individuos analizados, y la mayoría presentó al menos dos eventos de detención del crecimiento, entre los 4 y 6 años y medio. Ello indicaría que la niñez temprana sería sumamente estresante, principalmente durante aquella fase de crecimiento posterior al destete; probablemente a partir de los cambios en la dieta fueron expuestos a déficits nutricionales y a enfermedades infecciosas que comprometieron su supervivencia. Las presencia de anemia megaloblástica producto de carencia de vitamina B12 y/o ácido fólico puede ser debido a dietas deficientes en estos micronutrientes o a efectos de infecciones reiteradas. Es importante destacar que los individuos que combinaron hiperostosis porótica e hipoplasia de esmalte murieron tempranamente, mientras que el individuo que sólo presentó hipoplasia de esmalte presenta la mayor edad de muerte de la muestra. Esto ha sido retomado y discutido por Armelagos y colaboradores (2009), donde plantean evidencias en apoyo a la hipótesis de que los individuos que presentan mayor número de hipoplasias mueren más tempranamente que aquellos que no presentan lesiones. Esta idea es explicada a partir de que los daños fisiológicos que experimentan las personas durante una fase tan crítica del desarrollo como es la infancia y niñez, tienen consecuencias negativas a lo largo de la vida adulta.

\section{Conclusiones}

Según los resultados obtenidos con estos estudios, puede comprobarse que las condiciones de vida en la ciudad de Mendoza no fueron del todo adecuadas para el sostenimiento de condiciones sanitarias mínimas, principalmente durante los primeros tiempos de contacto hispano-indígena. Los cuerpos pertenecientes a individuos de sectores bajos de la sociedad de la muestra estudiada (enterrados fuera del primer templo jesuita), demostrarían una expectativa de vida relativamente baja que no superaba los 35 años. Todos presentaron evidencias de estrés fisiológico, anemia, algunos muestran claras evidencias de procesos infecciosos activos, y otros probablemente también murieron por alguna enfermedad infecto-contagiosa que no alcanzó a dejar registro en el sistema óseo. Esto lo entendemos como evidencia de las dificultades para afrontar con éxito el impacto causado, sobre todo, por enfermedades trasladadas por los conquistadores.

Un único individuo de la muestra fue adscrito al siglo XVIII; en él la edad de muerte es mayor, presenta treponematosis con un avanzado grado de curación y ausencia de anemia. En este sentido pensamos que la ciudad operaba en sentido positivo para el sostenimiento de la vida en algunos individuos (evidentemente de sectores altos, si 
se toma en cuenta su lugar de entierro), la que se mantendría con mayor eficacia que en sectores con mayores recursos socio-económicos. Este dato, sería consistente con las tendencias de crecimiento económico que, entre final del siglo XVII e inicios del XVIII, adquiere Mendoza (Prieto 2000).

Por otra parte, los resultados de las hipoplasias de esmalte indican que todos los individuos de la muestra aquí estudiada padecieron episodios de estrés recurrentes durante la segunda infancia, es decir entre los 3 a 6 años de edad, lo cual marca la posibilidad de haber padecido enfermedades infecciosas, gastrointestinales y/o deficiencias en los nutrientes, durante los primeros años de vida. Este dato cobra consistencia con la alta frecuencia de inhumaciones infantiles que estamos detectando en excavaciones de otros templos, como el de San Francisco-La Caridad o La Merced de la misma ciudad de Mendoza (Chiavazza et al. 2013; Mansegosa y Chiavazza 2010).

Entendemos que, con este análisis, queda evidenciado el gran potencial que poseen los estudios bioarqueológicos, como complemento imprescindible de ser articulado en los estudios histórico-sociales de procesos urbanos coloniales americanos.

Agradecimientos: Al equipo del Centro de Investigaciones Ruinas de San Francisco (Área Fundacional, Municipalidad de Mendoza), a la Municipalidad de Mendoza, a la Secretaría de Ciencia Técnica y Posgrado (UNCuyo, Mendoza) y CONICET. Al Dr. Luis Bosio por el reconocimiento de la patología treponematosis.

\section{Referencias bibliográficas}

Armelagos George J., Alan H. Goodman, Kristin N. Harper y Michael L. Blakey

2009 «Enamel Hypoplasia and Early Mortality: Bioarcheological Support for the Barker Hypothesis». Evolutionary Anthropology 18 (6): 261-271.

BARRIENTOS, Gustavo

1999 «Metodología de análisis de hipoplasia del esmalte dental aplicada al estudio de poblaciones prehispánicas del sudeste de la Región Pampeana». Revista Argentina de Antropología Biológica 2 (1): 307-322.

Barrientos, Gustavo, Rafael Goñı, Atilio F. Zangrando, Mariano del Papa, Solana García Guraieb, María Julia Arregui y Carla Negro

2007 «Human Taphonomy in Southern Patagonia: A View from the Salitroso Lake Basin (Santa Cruz, Argentina)», en Taphonomy and Zooarchaeology in Argentina, Ana María Gutiérrez, Laura Miotti, Gustavo Barrientos, Guillermo Mengoni y Mónica Salemme, eds., pp. 187-201. BAR International Series 1601. Oxford: Archaeopress.

BAss, William M.

1987 Human Osteology: A Laboratory and Field Manual. Special Publication 2. Springfield: Missouri Archaeological Society.

Buikstra, Jane E. y Douglas H. Ubelaker, eds.

1994 Standards for Data Collection from Human Skeletal Remains: Proceedings of a Seminar at the Field Museum of Natural History Organized by Jonathan Haas. Research Series No 44. Fayetteville: Arkansas Archaeological Survey. 
Byers, Steven N.

2002 Introduction to Forensic Anthropology. Boston: Allyn and Bacon.

Chiavazza, Horacio

2005 Los templos coloniales como estructuras funerarias. Arqueología en la iglesia Jesuita de Mendoza. BAR International Series S1388. Oxford: John and Erica Hedges Ltd. BAR.

2008 «Bases teóricas para el análisis arqueológico de la espacialidad religiosa y los procesos de transformación cultural en la ciudad de Mendoza durante la colonia». Revista de Arqueología Americana 25: 225-244.

Chiavazza, Horacio y Luis MafFerRa

2007 Estado de las investigaciones arqueobotánicas en Mendoza y sus implicancias en la arqueología histórica. Revista de Arqueología Histórica Latinoamericana y Argentina 1: 127-152.

Chiavazza Horacio, Alejandro Gámez Mendoza y Vanesa Guajardo

2013 «Ritos, cuerpos y lugares: análisis social desde un espacio religioso colonial (el templo de La Merced, Mendoza)», en XVII Congreso Nacional de Arqueología Argentina, J. Roberto Bárcena y Sergio E. Martín, eds., p. 381 La Rioja: Universidad Nacional de La Rioja.

ConI, Emilio R.

1897 Saneamiento de la provincia de Mendoza. Buenos Aires: Imprenta P.E. Coni.

Goodman, Alan H. y Jerome C. Rose

1991 «Dental Enamel Hypoplasias as Indicators of Nutritional Status», en Advances in Dental Anthropology, Mark A. Kelley y Clark S. Larsen, eds., pp. 279-292. Nueva York: Wiley-Liss Inc.

Hillson, Simon W.

1996 Dental Anthropology. Cambridge: Cambridge University Press.

LARSEN, Clark Spencer

1997 Bioarchaeology: Interpreting Behavior from the Human Skeleton. Cambridge: Cambridge University Press.

Lovejoy, Owen C., Richard S. Meindl, Thomas P. Pryzbeck y Robert P. Mensforth

1985 «Chronological Metamorphosis of the Auricular Surface of the Ilium: A New Method for the Determination of Adult Skeletal Age at Death». American Journal of Physical Anthropology 68 (1): 15-28.

MANSEgosa, Daniela

2010 «Estudios bioarqueológicos en un templo colonial de Mendoza: La Caridad», en XVII Congreso Nacional de Arqueología Argentina, Tomo 5, pp. 1777-1782. Mendoza: Facultad de Filosofía y Letras, Universidad Nacional Cuyo e INCIHUSA CCT Mendoza.

Mansegosa, Daniela y Horacio Chiavazza

2010 «Consecuencias del proceso de colonización en la salud de la población urbana de Mendoza (Argentina): un estudio desde evidencias paleopatológicas (siglos XVIII-XIX)». Revista Española de Paleopatología 8: 1-18.

Mansegosa, Daniela y Sebastián GiannotTi

2012 «Procesos analíticos para la elaboración del perfil de mortalidad de las series es- 
queletarias del punto arqueológico La Caridad (Área Fundacional de Mendoza)». Ponencia presentada en $V$ Jornadas Arqueológicas Cuyanas. INCIHUSA-CONICET. Mendoza.

Mansegosa Daniela, Alejandro Gámez Mendoza y Sebastián Giannotti

2013 «Análisis paleodemográficos y salubridad general de los grupos sociales marginados en una sociedad estamental a través del PA La Caridad (Mendoza). El Atlántico como puente en el proceso histórico: Mendoza y Canarias». Ponencia presentada en XVIII Congreso Nacional de Arqueología Argentina. Universidad Nacional de La Rioja.

MeInDL, Richard S. y Owen C. Lovejoy

1985 «Ectocranial Suture Closure: A Revised Method for the Determination of Skeletal Age at Death Based on the Lateral Anterior Sutures». American Journal of Physical Anthropology 68 (1): 57-66.

MENSFORTH, Robert

1991 «Paleoepidemiology of Porotic Hyperostosis in the Libben and BT-5 Skeletal Populations». Kirtlandia 46: 1-47.

Morales Juan, Eduardo Pena y Nidia Ferrer de Solti

2004 «Enfermedades de transmisión sexual», en Enfermedades infecciosas, J. Gorodner, ed., pp. 497-500. Rosario: Editorial Corpus.

ORTNER, Donald J.

2003 Identification of Pathological Conditions in Human Skeletal Remains, $2^{\mathrm{a}}$ ed. Amsterdam: Academic Press.

OrTner, Donald J. y Walter G.J. Putschar

1981 Identification of Pathological Conditions in Human Skeletal Remains. Smithsonian Contributions to Anthropology 28. Washington D.C.: Smithsonian Institution Press.

Prieto, M $\mathrm{M}^{\mathrm{a}}$ del Rosario

2000 Formación y consolidación de la sociedad en un área marginal del Reino de Chile [1983]. Tesis doctoral. Tirada especial de Anales de Arqueología y Etnología. Facultad de Filosofía y Letras. Universidad Nacional de Cuyo.

SCHEUER, Louise y Sue BLACK

2004 The Juvenile Skeleton. Londres: Elsevier Academic Press.

SchutKowski, Holger

1993 «Sex Determination of Infant and Juvenile Skeletons: I. Morphognostic Features». American Journal of Physical Anthropology 90 (2): 199-205.

Semorile, Adolfo, Elvira Martin de Codoni, Celia Cano Rossini, Marcos Puig Lopez y

Salvador LARIA

1988 Historia de la medicina en Mendoza. 2 Vols. Mendoza: Edición del autor.

ToDD, Wingate T.

1921 «Age Changes in the Pubic Bone». American Journal of Physical Anthropology 4 (1): $1-70$.

UBELAKER, Douglas H.

1989 Human Skeletal Remains: Excavation, Analysis, Interpretation. $2^{\mathrm{a}}$ ed. Washington D.C.: Taraxacum. 
Walker Phillip L., Rhonda R. Bathurst, Rebecca Richman, Thor GJerdrum y Valerie A. ANDRUSHKO

2009 «The Causes of Porotic Hyperostosis and Cribra Orbitalia: A Reappraisal of the Iron-Deficiency-Anemia Hypothesis». American Journal of Physical Anthropology 139 (2): 109-125.

WEAVER, David S.

1980 «Sex Differences in the Ilia of a Known Sex and Age Sample of Fetal and Infant Skeletons». American Journal of Physical Anthropology 52 (2): 191-195. 